\title{
ASYMPTOTIC GLUON SHADOWING
}

\author{
S. LIUTI \\ Institute of Nuclear and Particle Physics, University of Virginia, \\ McCormick Road, Charlottesville, Virginia 22901, USA \\ and \\ INFN-Sezione Roma Tre, Dipartimento di Fisica E. Amaldi, \\ Via Vasca Navale, 84. 00146 Roma, Italy. \\ E-mail: sl4y@virginia.edu \\ F. CANO \\ Dipartmento di Fisica, Universitá di Trento, \\ O38050 Povo, Trento, Italy \\ E-mail: cano@science.unitn.it
}

We examine the low Bjorken $x$ gluon distribution in nuclei in the asymptotic region.

\section{Introduction}

Nuclear shadowing or the depletion at low Bjorken $x$ of the nuclear Deep Inelastic (DI) structure function, $F_{2}^{A}$, with respect to the nucleon one, $F_{2}^{N}$, has been observed in a number of experiments (seet and references therein). Assuming the universality of parton distributions in nuclei, one expects nuclear shadowing to be present in other high energy processes as well, such as Drell-Yan pair, $J / \psi$ and $\Upsilon$ production in lepton-nucleus, hadron-nucleus and nucleus-nucleus collisions. In particular nuclear shadowing might be concurring with the other mechanisms among which quark-gluon plasma formation, that result in a depletion of the observed cross sections for these processes 2 . A quantitative understanding of both the $x$ and $Q^{2}$ dependences of the nuclear parton distributions at low and very low $x$ is therefore a necessary step for interpreting the outcome of future experiments at RHIC and at the LHC. Recent calculations rely on non-perturbative models for the puclear parton distributions at a given (low) scale, $Q_{o}^{2}$, combined with DGLAP 4 perturbative evolution. They are all therefore affected by the uncertainty in the initial parton distributions and, in particular, in the gluon distribution which governs evolution at low $x$, and which is poorly known experimentally. Moreover, evolution to moderate values of $Q^{2}\left(Q^{2} \approx 10 \mathrm{GeV}^{2}\right)$ depends even more strongly, by the value of $Q_{o}^{2}$ itself which, if varied within the range, $Q_{o}^{2}=0.8-$ few $\mathrm{GeV}^{2}$ can lead to completely different evolution patterns for the shadowing of both the structure functions and the gluon distribution. These simple facts hamper the possibility of pre- 
dicting the $Q^{2}$ dependence of nuclear shadowing although its behavior within pQCD is quite well understood and it can be predicted accurately for each choice of initial conditions.

In this paper we suggest an avenue for dealing with the model dependence associated with the initial conditions of perturbative evolution, which is based on a study of the low $x$ and high $Q^{2}$ asymptotic behavior of the shadowing ratios $R_{G}=G_{A} / G_{N}$ and $R_{F}=F_{2}^{A} / F_{2}^{N}, G_{N(A)}$ and $F_{2}^{N(A)}$ being the gluon distributions and the Deep Inelastic (DI) structure function in a nucleon, $(N)$, and in an isoscalar nucleus, $(A)$, respectively. In the asymptotic regime defined by $Q^{2} \gg Q_{o}^{2}$ and $x \ll x_{o}, x_{o} \leq 0.1$, the Double Logarithmic Approximation (DLA) to pQCD evolution applies 5. The proton structure function data analyzed recently at HERA 6 G he been proven 6 after the suggestion of Refll, to evolve according to DLA. The key test is to show that the data obey Double Asymtptotic Scaling (DAS) in the variables $\rho=\gamma\left(\left(Y-Y_{o}\right) / \xi\right)^{1 / 2}$ and $\sigma=\gamma^{-1}\left(\left(Y-Y_{o}\right) \xi\right)^{1 / 2}, \gamma=6 /\left(33-2 N_{f}\right)^{1 / 2}$, $Y=\ln 1 / x, \xi=\gamma^{2} \ln \left(\ln Q^{2} / \Lambda_{Q C D}^{2} / \ln Q_{o}^{2} / \Lambda_{Q C D}^{2}\right)$. Violations from DAS (other than due to the fact that the data lie in a pre-asymptotic region or to NLO DGLAP corrections Ab $_{b}$ ) would signal either the onset of other approximations such as leading-(next-to-leading)-log in $1 / x, L L_{x}\left(N L_{x}\right)$ resummation, or the beginning of parton saturation. Here we wish to apply a DAS type of analysis to nuclear DI scattering.

As a first step we show that if evolution proceeds through ordinary asymptotic pQCD evolution equations in nuclei as well as in a free nucleon, the ratios $R_{F}$ and $R_{G}$ become a function of $\rho$ only, which is per se a model independent result. We use this result as a basis for exploring the origin of scaling violations in nuclei. In nuclei in fact, the asymptotic regime is entered in principle at different values of $x_{o}^{A}$ and $Q_{o, 4}^{2}$ than in the proton. In particular Unitarity Shadowing Corrections (USC) 9.10,11.12 are expected to affect evolution at $x_{o}^{A}>x_{o}$ because of the increase of the gluon density in a nucleus due to the overlapping of nucleons in the longitudinal direction. On a more speculative basis one might also expect the transition to the $\ln (1 / x)$ resummation to appear in a different regime. In our approach such questions can be addressed systematically as they introduce specific scaling violations from the DLA result, appearing as a $\sigma$ dependence in the ratios $R_{G}$ and $R_{F}$.

In summary, although it is technically predictable that in a proton at very low values of $x$ and sufficiently large $Q^{2}$, i.e. deeply in the asymptotic region, DGLAP evolution and the DLA should break down and give way to $\ln (1 / x)$ summation and to USC, it is still a major task to be able to pinpoint where and if the transition from the different regimes is going to take place in the kinematical regimes currently under exploration. Our goal is to obtain 
some new insight by using nuclear targets where the asymptotic regime can in principle be reached at larger $x$. As a by-product we obtain quantitative predictions for RHIC and the LHC.

\section{DGLAP Evolution in Nuclei}

We first summarize results for ordinary DGLAP evolution applied to the nuclear ratios at low $x$, assuming that the proton and the nuclear distributions evolve along similar paths. As it is well known evolution is driven by the gluon distribution which dominates over the sea quarks one and one can predict the behavior of the shadowing ratios, $R_{G}$ and $R_{F}$ with $Q^{2}$ :

$$
\begin{aligned}
\frac{\partial R_{G}}{\partial \ln Q^{2}} \simeq \int_{x}^{1} P_{G G}\left(\frac{x}{y}, \alpha_{S}\left(Q^{2}\right)\right) \frac{G_{N}\left(y, Q^{2}\right)}{G_{N}\left(x, Q^{2}\right)} \\
\quad \times\left[R_{G}\left(y, Q^{2}\right)-R_{G}\left(x, Q^{2}\right)\right] \frac{d y}{y} \\
\propto \frac{\partial G_{A}\left(x, Q^{2}\right) / \partial \ln Q^{2}}{\partial G_{N}\left(x, Q^{2}\right) / \partial \ln Q^{2}}-R_{G}\left(x, Q^{2}\right), \\
\frac{\partial R_{F}}{\partial \ln Q^{2}} \simeq \int_{x}^{1} P_{q G}\left(\frac{x}{y}, \alpha_{S}\left(Q^{2}\right)\right) \frac{G_{N}\left(y, Q^{2}\right)}{\Sigma_{N}\left(x, Q^{2}\right)} \\
\times\left[R_{G}\left(y, Q^{2}\right)-R_{F}\left(x, Q^{2}\right)\right] \frac{d y}{y},
\end{aligned}
$$

where we have disregarded the sea quarks distribution on the r.h.s. of the coupled DGLAP evolution equations; $P_{q G}$ and $P_{G G}$ are the splitting functions evaluated at NLO; and we used the approximation $F_{2}^{N(A)} \approx 5 / 18 \Sigma_{N(A)}$. Eqs.(1) and (2) show that the $Q^{2}$ dependence of $R_{G}$ and $R_{F}$ is regulated by a subtle balance involving the parton distributions and the ratios themselves 13. The following predictions can be made for the ratios $R_{G}$ and $R_{F}$ : If, as predicted by current non-perturbative shadowing models $R_{G}$ is a growing function of $x$, then it also grows with $Q^{2}$, the r.h.s. of Eq.(1) being positive $(y \geq x)$. On the other side, defining $R_{G}\left(x, Q_{o}^{2}\right) \equiv R_{G}^{o}$ and $R_{F}\left(x, Q_{o}^{2}\right) \equiv R_{F}^{o}$, one obtains two opposite behaviors for $R_{F}$ namely: i) if $R_{F}^{o}<R_{G}^{o}$, then $R_{F}$ grows with $Q^{2}$; ii) if instead $R_{F}^{o}>R_{G}^{o}$, then $R_{F}$ initially decreases with $Q^{2}$ until it reaches the value of $R_{G}^{o}$ and it subsequently starts increasing along with $R_{G}$. 0 The "rate" of change with $Q^{2}$ is governed by the ratios $G_{N}(y) / G_{N}(x)$ and $G_{N}(y) / \Sigma_{N}(x)$, at $Q^{2}=Q_{o}^{2}$, respectively. If $Q_{o}^{2} \leq 1 \mathrm{GeV}^{2}$, then a rapid

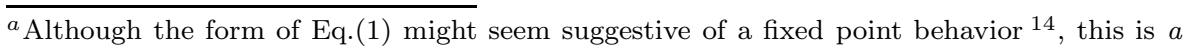
priori not the case, since the quantity $\partial G_{A}\left(x, Q^{2}\right) / \partial \ln Q^{2} / \partial G_{N}\left(x, Q^{2}\right) / \partial \ln Q^{2}$ depends on $Q^{2}$. 
evolution strongly reduces the shadowing in both $R_{G}$ and $R_{F}$, between $Q_{o}^{2}$ and $Q^{2} \approx 2-3 \mathrm{GeV}^{2}$. If on the contrary $Q_{o}^{2}$ ranges from 2 to $5 \mathrm{GeV}^{2}$ then the evolution is slower and shadowing remains large even at $Q^{2} \approx 10-100 \mathrm{GeV}^{2}$ (these results affect quarkonia production where $Q^{2} \equiv M_{J / \psi}^{2} \approx 10 \mathrm{GeV}^{2}$ and $\left.Q^{2} \equiv M_{\Upsilon}^{2} \approx 100 \mathrm{GeV}^{2}\right)$

\section{Double Asymptotic Scaling}

We now examine the nuclear DI structure function and gluon distribution in the asymptotic regime. The derivation of the equations of the DLA in a nucleus parallels the one for the proton, namely one first writes the LO DGLAP evolution equation for the gluon distribution in the limit $x \rightarrow 0$, in moments space (we have omitted the subscripts $N(A)$ unless necessary):

$$
\frac{\partial g\left(n, Q^{2}\right)}{\partial \ln Q^{2}}=\frac{\alpha_{s}\left(Q^{2}\right)}{2 \pi} \gamma_{G G}^{0}(n) g\left(n, Q^{2}\right),
$$

$\gamma_{g g}^{0}(n) \approx 2 C_{A} /(n-1)$ being the anomalous dimension in the limit $n \rightarrow 1$. Solutions in $\left(x, Q^{2}\right)$ are found by evaluating the anti-Mellin transform,

$$
\begin{aligned}
G\left(x, Q^{2}\right)= & \frac{1}{2 \pi i} \int_{C} \operatorname{dng}\left(n, Q_{0}^{2}\right) \\
& \exp \{(n-1) Y+\xi /(n-1)\},
\end{aligned}
$$

with the saddle point method. In Eq.(化) $g\left(n, Q_{0}^{2}\right)=\int_{0}^{1} d x x^{(n-1)} g\left(x, Q_{0}^{2}\right)$, $g\left(x, Q_{o}^{2}\right)$ being the inital gluon distribution, and $G\left(x, Q^{2}\right)=x g\left(x, Q^{2}\right)$. If one takes a "soft" initial condition such as, $G\left(x, Q_{0}^{2}\right) \approx A_{N} x^{-\lambda}, \lambda \approx 0$, and $Y$ and $\xi$ are both similarly large, then $g\left(n_{0}, Q_{0}^{2}\right) \approx A_{N} /(n-1)$, and the saddle point is: $n_{0}=1 /(2 \Delta Y)+\sqrt{1 /(4 \Delta Y)^{2}+\xi / \Delta Y} \approx \sqrt{1+\xi / \Delta Y}, \Delta Y=Y-Y_{o}$, $Y_{o}=\ln \left(1 / x_{o}\right)$, yielding:

$$
G\left(x, Q^{2}\right)=\sqrt{2 \pi}\left(\frac{\widetilde{g}\left(n_{o}, Q_{o}^{2}\right)}{\widetilde{g}^{\prime \prime}\left(n_{o}, Q_{o}^{2}\right)}\right)^{1 / 2} \widetilde{g}\left(n_{o}, Q^{2}\right)
$$

with $\widetilde{g}\left(n, Q^{2}\right)=g\left(n, Q^{2}\right) \exp [(n-1) Y]$. I In terms of the DAS variables, $\rho$ and $\sigma, n_{0}=1+\rho / \gamma^{2}$ and:

$$
G \equiv G^{D L A}\left(x, Q^{2}\right)=f(\rho, \sigma) \exp (2 \gamma \sigma),
$$

where $f(\rho, \sigma)$ is a function that depends on the explicit form of the initial conditions. The main result is that $\left.\ln \left(G^{D L A}\right) / f(\rho, \sigma)\right)$ is predicted to be linear

${ }^{b}$ We have omitted sub-leading corrections for simplicity. 
in $\sigma$, the slope being fixed by $\gamma$. A similar behavior is found for $F_{2}^{N}$ by solving the equation:

$$
\frac{\partial F_{2}^{N}\left(x, Q^{2}\right)}{\partial \ln Q^{2}}=\frac{2}{9} \frac{\alpha_{S}}{\pi} G\left(x, Q^{2}\right) .
$$

Deviations from DAS due to hard initial conditions i.e. when $\lambda \geq 0.2$ are technically obtained as follows: $g\left(n_{0}, Q_{0}^{2}\right) \approx A_{N} /(n-(\lambda+1))$ and a physical solution in the limit $Y \gg \xi$ is

$$
G\left(x, Q^{2}\right)=C / \lambda e^{\lambda\left(Y-Y_{o}\right)+\xi / \lambda},
$$

corresponding to the saddle point value, $n_{0}(\lambda)=1 / \Delta Y+\lambda+1$ (see also 6 , 1 ). Physically this behavior supports the appearance of USC which become more important just because of the steep rise at small $x$. The data of Refl favor the DAS scenario, whereas the more recent data at lower $x$ and $Q^{2} \mathrm{Z}$ are best interpreted in terms of USC 16 . In this context it is therefore important to study nuclear shadowing because, due to the $A$-dependent increase in gluon density, the physics we are interested in is expected to show up at larger $x$ values and because one can tune the variable $A$ in order to discriminate among models.

In a nucleus we make the two following assumptions for the asymptotic regime: i) the initial distributions are shadowed because of some non-perturbative mechanism, the evolution equations are not affected by the medium; ii) besides the non-perturbative shadowing the evolution equations have screening corrections at a larger $x_{A}$. The derivation of the ratio $R_{G}$ in the hypothesis $i$ ) yields:

$$
\begin{array}{r}
R_{G}^{A s y m}=\left[\frac{G_{A}\left(n_{o}, Q^{2}\right)}{G_{N}\left(n_{o}, Q^{2}\right)}\right]^{3 / 2}\left[\frac{G_{N}^{\prime \prime}\left(n_{o}, Q^{2}\right)}{G_{A}^{\prime \prime}\left(n_{o}, Q^{2}\right)}\right]^{1 / 2} \\
=f_{A}(\rho) \frac{\rho h_{N}^{(1)}(\rho)+\gamma^{2} \sigma h_{N}^{(2)}(\rho)}{\rho h_{A}^{(1)}(\rho)+\gamma^{2} \sigma h_{A}^{(2)}(\rho)}
\end{array}
$$

where $f_{A}(\rho)=G_{A}\left(n_{o}, Q_{o}^{2}\right) / G_{N}\left(n_{o}, Q_{o}^{2}\right)$, and the functions $h_{N(A)}^{(1,2)}$ are listed explicitely elsewhere. The main point is that the exponential terms appearing in $G^{D L A}$, Eq.(6) calculated for a nucleus and for a nucleon respectively, cancel exactly and the remaining dependence on $\sigma$ is small asymptotically. This scaling result (shown in Fig.1) will be our reference point.

The onset of a different evolution mechanism in the nucleus will appear as a $\sigma$-scaling violation modifying the exponential behavior of Eq.(5). In general one can write

$$
R_{G}=R_{G}^{A s y m}(\rho) \times \exp \left\{2 \gamma\left(\sigma_{A}-\sigma\right)\right\},
$$



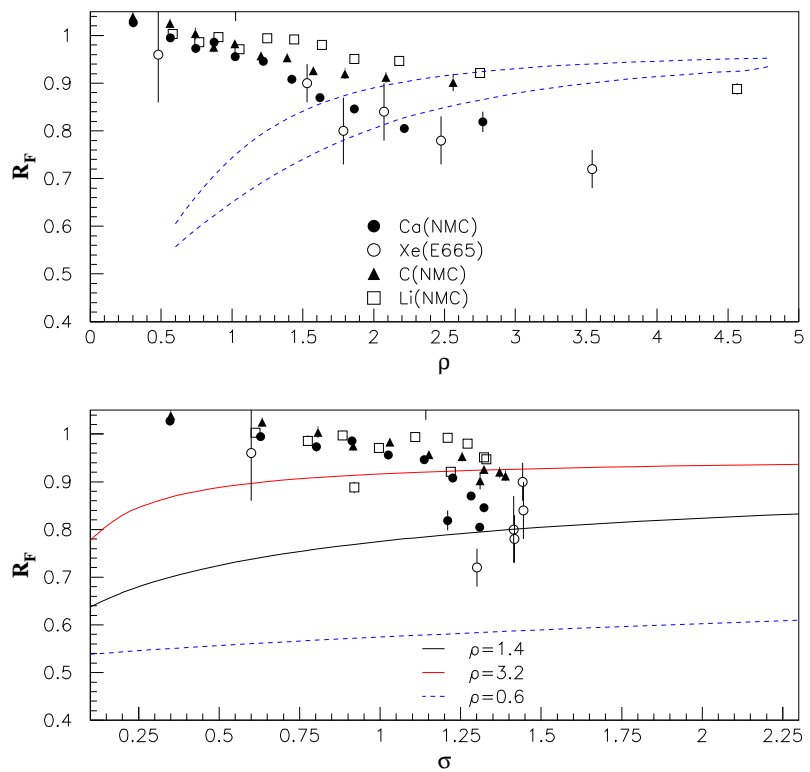

Figure 1: Nuclear structure fung ratios, $R_{F}$, vs. the DAS variables $\rho$ (top) and $\sigma$ (bottom). The theoretical curves 20 show the onset of scaling in $\sigma$. The non-pertopative

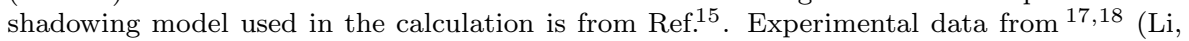
$\mathrm{C}, \mathrm{Ca})$ and $\mathrm{ES}(\mathrm{Xe})$.

where $R_{G}^{\text {Asym }}$ is the large $\sigma$ limit of Eq.(9). The form of $\sigma_{A} \equiv \sigma_{A}\left(x, Q^{2}\right)$ describes the approximation to pQCD evolution in a nucleus. In the case of a "hard pomeron" boundary conditions plus screening corrections 9 it is

$$
\sigma_{A}=D_{A}\left(x, Q^{2}\right)\left(\lambda \sigma \rho+\frac{\gamma^{2}}{\lambda} \frac{\sigma}{\rho}\right)
$$

$D_{A}$ being a damping correction (more details are going to be found in 20 ).

In conclusion, we have shown model independent predictions for gluon shadowing in nuclei in the asymptotic region. We have also outlined an approach for more detailed studies of the violations from DAS in nuclei as a signal of pQCD evolution mechanisms other than DGLAP or the DLA. Our calculations will be relevant in the regime accessible at future experiments at RHIC, LHC and possibly at the $e A$ project at DESY 21. 


\section{Acknowledgments}

The authors wish to thank the Institute of Nuclear and Particle Physics at the University of Virginia for financial support.

\section{References}

[1] M. Arneodo, Phys. Rep. 240 (1994) 301.

[2] Zi-wei Lin and M. Gyulassy, Phys.Rev.Lett. 77 (1996) 1222.

[3] S. Liuti and R. Vogt, Phys. Rev. C51 (1995) 2244.

[4] L.V. Gribov and Lipatov, Yad.Fiz. 20 (1975) 181; G. Altarelli and G. Parisi, Nucl.Phys. B126 (1977) 298; Dokshitzer, Sov.Phys.JETP 46 (1977) 641.

[5] A. De Rujula et al., Phys. Rev. D10 (1974) 1649.

[6] H1 Collaboration, T.Ahmed et al., Nucl.Phys. B439 (1995) 471; C. Adloff et al., Nucl.Phys. B497 (1997) 3; S. Aid et al., Nucl.Phys. B470 (1996), 3.

[7] ZEUS Collaboration, M. Derrick et al., Z.Phys. C69 (1996) 607; J. Breitweg et al., Phys.Lett. B407 (1997) 432

[8] R. Ball and S. Forte, Phys. Lett. B335 (1994) 77; ibid B336 (1994) 77; Nucl. Phys. Proc. Suppl. 54A (1997) 163.

[9] L.V. Gribov, E.M. Levin and M.G. Ryskin, Phys. Rep. 100 (1983) 1.

[10] A.H. Mueller and J. Qiu, Nucl. Phys. B268 (1986) 427. (1994) 44.

[11] J.C. Collins and I. Kwiecinski, Nucl. Phys. B335 (1990) 89.

[12] K. Eskola, J. Qiu and X. Wang, Phys.Rev.Lett. 72 (1994) 36.

[13] J. Qiu, Nucl. Phys. B291 (1987) 746.

[14] Z. Huang, H.J. Lu and I. Sarcevic, Nucl. Phys. A637 (1998) 79.

[15] L.L. Frankfurt and M.I. Strikman, Nucl. Phys. B316 (1989) 340.

[16] E. Gotsman, E.Levin, U.Maor and E. Naftali, Nucl. Phys. B (1999), hep-ph/9808257.

[17] M. Arneodo et al., Nucl. Phys. B441 (1995) 12.

[18] P. Amaudruz et al., Z. Phys. C53 (1992) 73.

[19] M.R. Adams et al., Phys. Lett. B287 (1992) 375; Phys. Rev. Lett. 68 (1992) 3266.

[20] F. Cano and S. Liuti, in preparation.

[21] Workshop on Physics with HERA as eA Collider, DESY, Hamburg, 2526 May, 1999, C99/05/95. 